\title{
Neutrino Vertex Reconstruction in South Pole Ice
}

\author{
M. Beheler-Amass ${ }^{1}$, A. Karle ${ }^{1}$, J. L. Kelley ${ }^{1, \star}$, and M.-Y. Lu ${ }^{1}$ for the ARA Collaboration \\ ${ }^{1}$ WIPAC / Dept. of Physics, Univ. of Wisconsin-Madison, Madison, WI, USA
}

\begin{abstract}
Reconstruction of potential ultra-high-energy (UHE) neutrino events at the Askaryan Radio Array (ARA) is complicated by the variable index of refraction of South Pole ice, leading to curved radio signal paths from the interaction vertex. Using a spline table framework for fast raytracing approximation, we perform a GPU-accelerated interferometric reconstruction of the event vertex. We also demonstrate how use of both direct and reflected/refracted radio signals can allow reconstruction of the distance to the interaction vertex, an important step towards neutrino energy reconstruction.
\end{abstract}

\section{Introduction}

The Askaryan Radio Array (ARA) is an ultra-high-energy neutrino detector under construction at the South Pole [1]. Five detector stations search for the broadband radio-frequency Askaryan emission from neutrino interactions in the ice. Each station consists of a cluster of vertically- and horizontallypolarized antennas deployed in dry boreholes up to $200 \mathrm{~m}$ deep in the ice.

Reconstruction of the position of the neutrino interaction vertex is the first step in the determination of neutrino energy and direction. The direction from the detector station to the interaction vertex can be reconstructed from the radio pulse arrival times at the antennas. This direction is different from the incoming neutrino direction because of the Cherenkov cone geometry of the Askaryan emission. Because the density of the ice decreases in the transition to the compacted snow "firn" region at the surface, radio signal paths are not straight lines and can also reflect or refract off the surface, meaning the pulse can possibly be received twice (Fig. 1). If observed, the second pulse provides additional information that can be used in vertex reconstruction. More realistic firn models including discontinuities and layering introduce more propagation modes [2]; these are not considered here.

\section{Interferometric Event Reconstruction}

An accurate neutrino interaction vertex reconstruction requires arrival times of the radio signals at the antennas that take into account the curved paths through the ice. A full raytracing calculation from a large number of hypothesized vertices can be prohibitively slow, so we have developed an approximation technique that uses precalculated raytracing tables interpolated with multi-dimensional B-splines [3]. To reconstruct the direction from the station to the neutrino interaction vertex, we form an interferometric skymap of the total cross-correlation of pairs of antenna waveforms, given the arrival time differences at each pair from the spline lookup. The reconstruction has been parallelized

^e-mail: jkelley@icecube.wisc.edu 


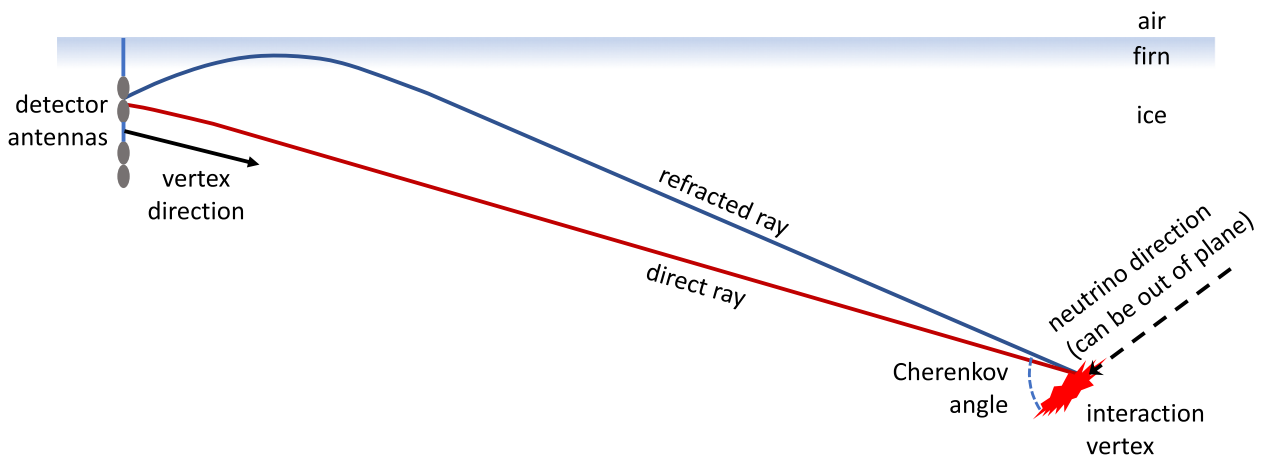

Figure 1. Geometry of neutrino vertex reconstruction in ice, with both direct and refracted signal paths from the interaction vertex to the detector antennas (not to scale). The neutrino direction vector is shown in the plane here for simplicity; in general this is not the case.

for graphics processing units (GPUs) to further improve performance. For simulated neutrinos of $10^{18}$ $\mathrm{eV}$ energy, the vertex angular resolution is approximately $0.3^{\circ}$ in zenith and azimuth.

In order to estimate neutrino energy, we are also interested in the distance from the center of the detector station to the interaction vertex. We expand the reconstruction to form skymaps over a range of reasonable distance hypotheses, and then consider the maximum cross-correlation pixel in this entire set of skymaps. We find very limited distance resolution for simulated events using this method. This is not unexpected, since by using timing information, we are attempting to measure the curvature of the wavefront, a much more difficult task than simply the wavefront arrival direction.

\section{Vertex Reconstruction with Multipath Signals}

Both direct and reflected signals can be observed from deep, distant calibration pulsers installed on two of the strings of the IceCube Neutrino Observatory [4]. By separating the pulses into waveform "snippets" and adding them to the cross-correlation reconstruction, the 4-km distance to the deep pulser can be reconstructed to within $13 \%$. This includes a systematic offset; the statistical spread is significantly less.

To understand both the power and limitations of this method, we consider a simple, 2D geometric example using two idealized antennas and timing from raytracing calculations. First, the arrival time difference of the direct pulses in two antennas specifies a single direct ray along which the neutrino interaction vertex lies, by determining the angle of the incoming signal. Then the time difference between the direct and reflected pulse in a single antenna specifies the distance along this ray of the vertex. Figure 2 shows how this direct-to-reflected time difference changes as one moves along the allowed ray for an example vertex location. Because the reflected path becomes smaller for shallower antennas,

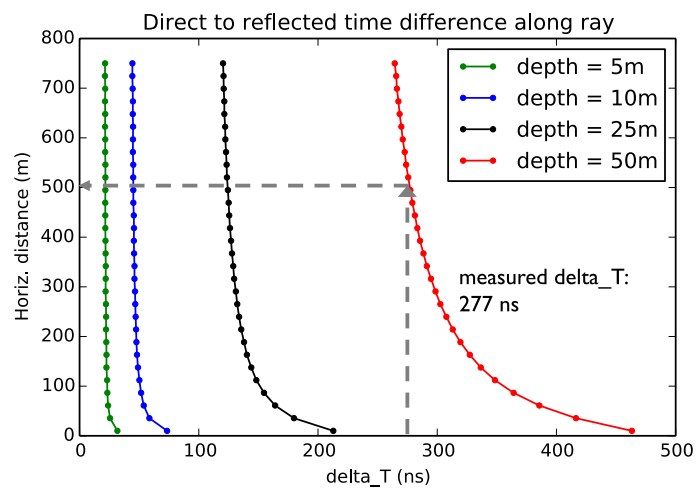

Figure 2. Example lookup of distance to interaction vertex based on time difference between direct and reflected rays, for different antenna depths. 
the time resolution required to measure the vertex also becomes more stringent - the slope of the curve in Fig. 2 is a mapping from timing resolution to distance resolution. For this particular idealized example, to achieve $10 \%$ distance resolution, a timing resolution of $60 \mathrm{ps}$ is required for $5 \mathrm{~m}$-deep antennas, and resolution of $0.75 \mathrm{~ns}$ is required for $25 \mathrm{~m}$-deep antennas.

A realistic estimate of double-pulse efficiency must take into account the Cherenkov emission pattern and propagation of the Askaryan signal, not just the raytracing geometry. We have performed a full simulation of neutrino events at various energies for stations at a range of depths. The initial step in determining the efficiency is identifying double-pulse waveforms in the simulated data. Using the autocorrelation of the waveform, we search for a secondary maximum after the point where the slope of the autocorrelation first becomes zero (Fig. 3). We then consider the amplitude of the secondary autocorrelation peak and the "baseline" level of the autocorrelation and perform a 2D cut to select double-pulse events from the background of thermal noise and single pulses.

Using this selection, we calculate the fraction of double-pulse events as a function of station depth and neutrino energy, where a double-pulse event must contain at least one waveform selected by the previous autocorrelation algorithm. Fig. 4 shows the preliminary double-pulse efficiency at trigger level. At $10^{18} \mathrm{eV}$ neutrino energies, approximately $40 \%$ of events are identified as double-pulse at a station depth of $25 \mathrm{~m}$, while at $200 \mathrm{~m}, 10 \%$ of events contain a double pulse.

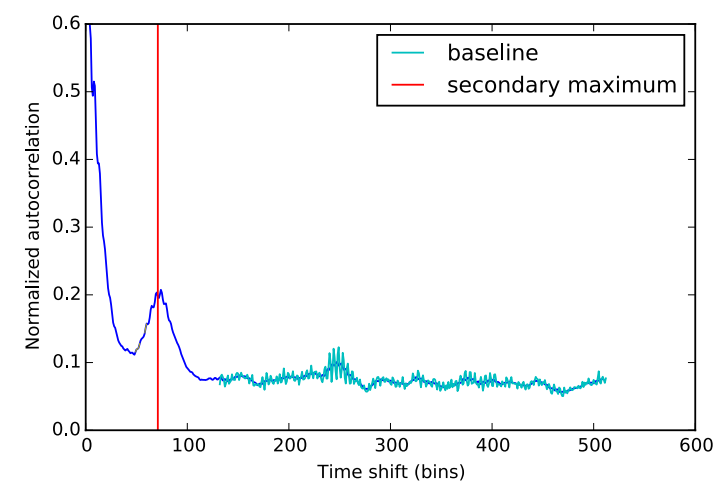

Figure 3. Smoothed autocorrelation of a simulated doublepulse waveform. The secondary pulse is evident as a secondary maximum at non-zero time shift.

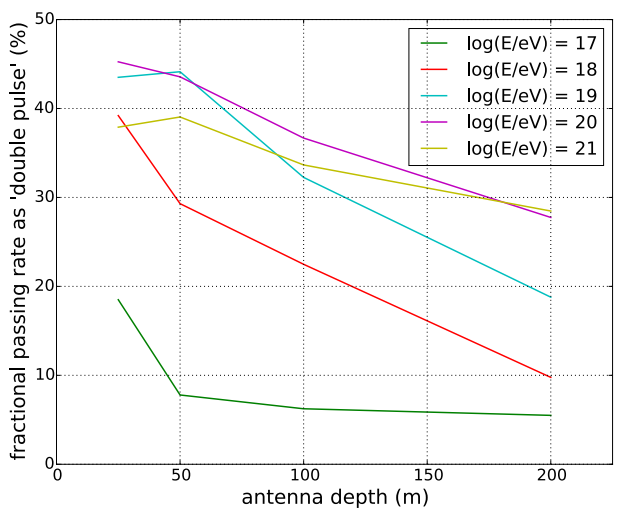

Figure 4. Fraction of double-pulse events at ARA trigger level, as a function of neutrino energy and station depth (preliminary).

Using this framework for identifying double pulses, we plan to develop a general vertex reconstruction method using both direct and reflected pulse snippets, to quantify the distance resolution in a full detector simulation chain. We also plan to continue to evaluate the depth dependence. Given that double-pulse efficiency decreases with depth, but distance resolution should improve with depth, we expect to find some optimum antenna depth range when taking both into account. This will be relevant for future detector designs.

\section{References}

[1] P. Allison et al. (ARA Collaboration), Phys. Rev. D 93, 082003 (2016)

[2] S. Buitink, these proceedings

[3] M. Beheler-Amass et al. (ARA Collaboration), Proc. 35 ${ }^{\text {th }}$ ICRC, PoS(ICRC2017) 1054 (2017)

[4] D. Seckel et al. (ARA Collaboration), Proc. $35^{\text {th }}$ ICRC, PoS(ICRC2017) 1030 (2017) 